Supporting information

\title{
Bisphosphonate stabilised calcium phosphate nanoparticles for effective delivery of plasmid DNA to Macrophages
}

Bing Sun ${ }^{1}$, Marianne Gillard ${ }^{1}$, Yanheng Wu ${ }^{1}$, Peihong Wu ${ }^{2}$, Zhi Ping Xu ${ }^{1} \bowtie$ Wenyi Gu $^{1} \bowtie$

1. Australian Institute for Bioengineering and Nanotechnology, University of Queensland, St Lucia, QLD 4072, Australia

2. State Key Laboratory of Oncology in South China, Sun Yat-Sen University, Guangzhou China;

$\bowtie$ : corresponding authors:

Address: Australian Institute for Bioengineering and Nanotechnology (Building 75), The University of Queensland, Cooper Rd., St Lucia, Brisbane QLD 4072, Australia.

ZX: email: gordonxu@uq.edu.au, Tel: 61-7-334 63809; WG: email: w.gu@uq.edu.au, Tel:

$61-7-33463814$ 
1 Synthesis and characterization of BCP NPs

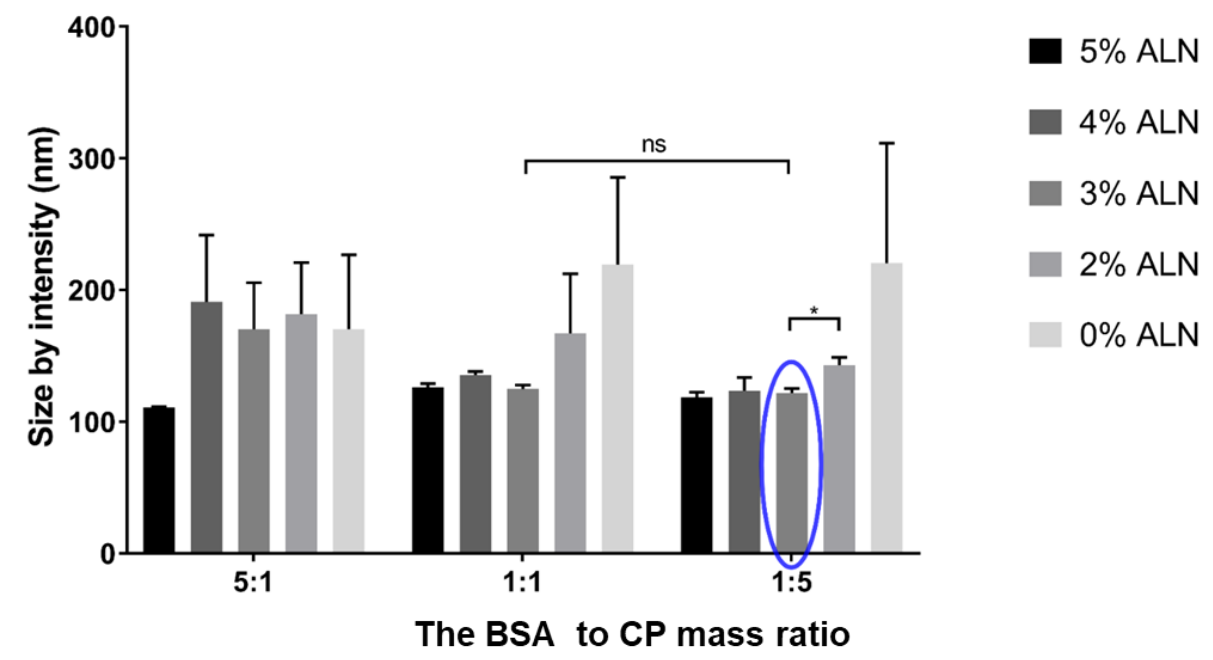

Figure S1. The size of CP NPs with different ALN and BSA coating ratio.

Table S1. The PdI of CP NPs with different ALN and BSA coating ratio.

\begin{tabular}{cccc}
\hline & \multicolumn{3}{c}{ BSA:CP ratio } \\
\cline { 2 - 4 } & $5: 1$ & $1: 1$ & $1: 5$ \\
\hline 5\% ALN & $0.229 \pm 0.006$ & $0.196 \pm 0.009$ & $0.231 \pm 0.015$ \\
3\% ALN & $0.229 \pm 0.013$ & $0.210 \pm 0.025$ & $0.247 \pm 0.008$ \\
$2 \%$ ALN & $0.208 \pm 0.017$ & $0.247 \pm 0.007$ & $0.200 \pm 0.013$ \\
$0 \%$ ALN & $0.229 \pm 0.008$ & $0.223 \pm 0.008$ & $0.208 \pm 0.021$ \\
& $0.235 \pm 0.012$ & $0.254 \pm 0.013$ & $0.370 \pm 0.019$ \\
\hline
\end{tabular}
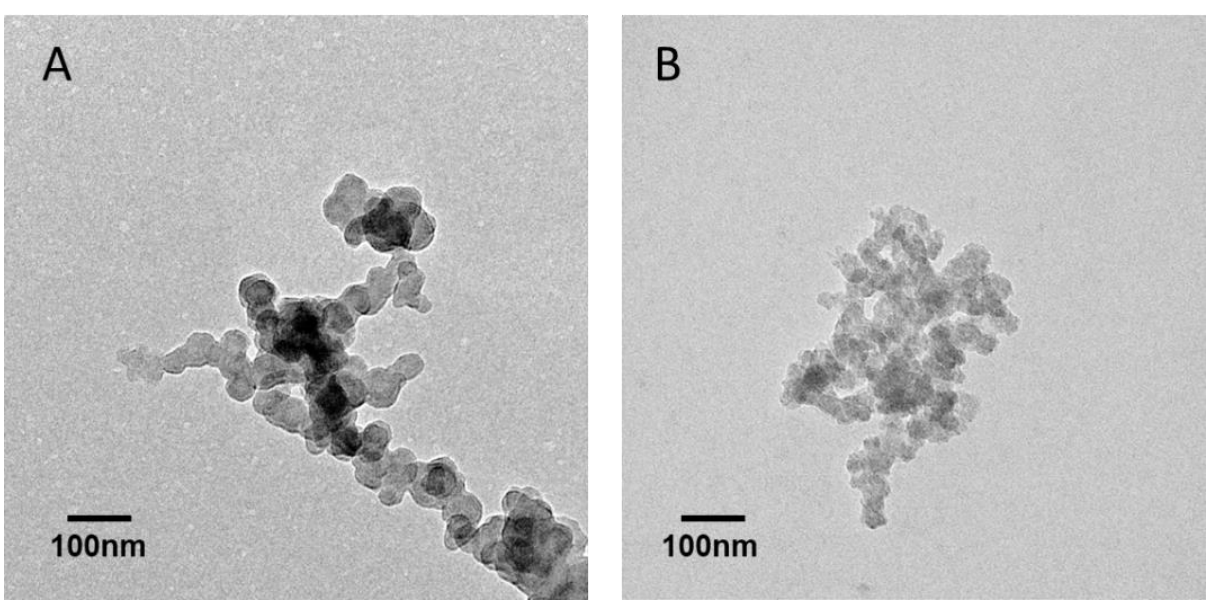

Figure S2. The TEM images of CP NPs (A) and CP-pEGFP NPs (B) 


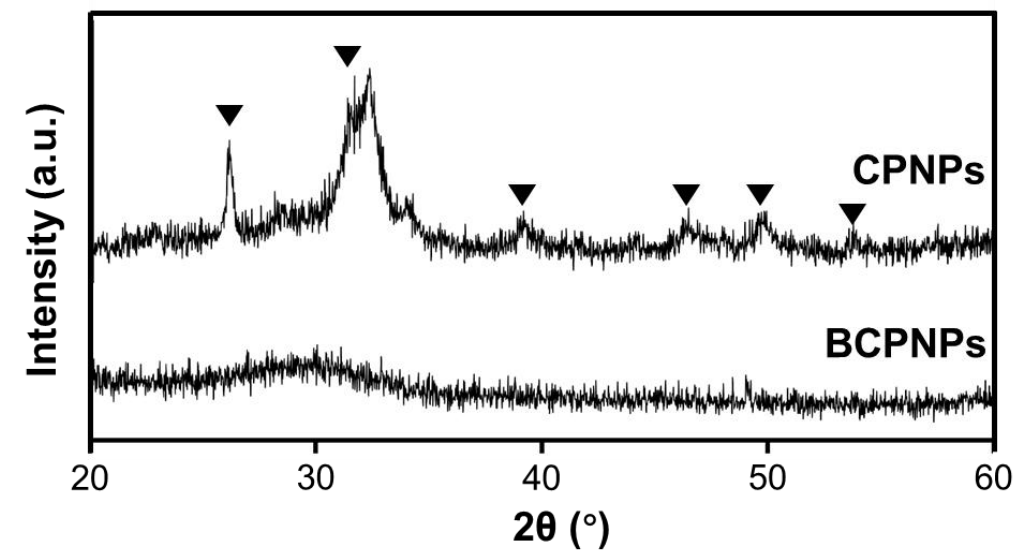

Figure S3. The XRD spectrum of CPNPs and BCPNPs. The BCPNPs show an amorphous pattern while CPNPs show a crystalline apatite pattern.

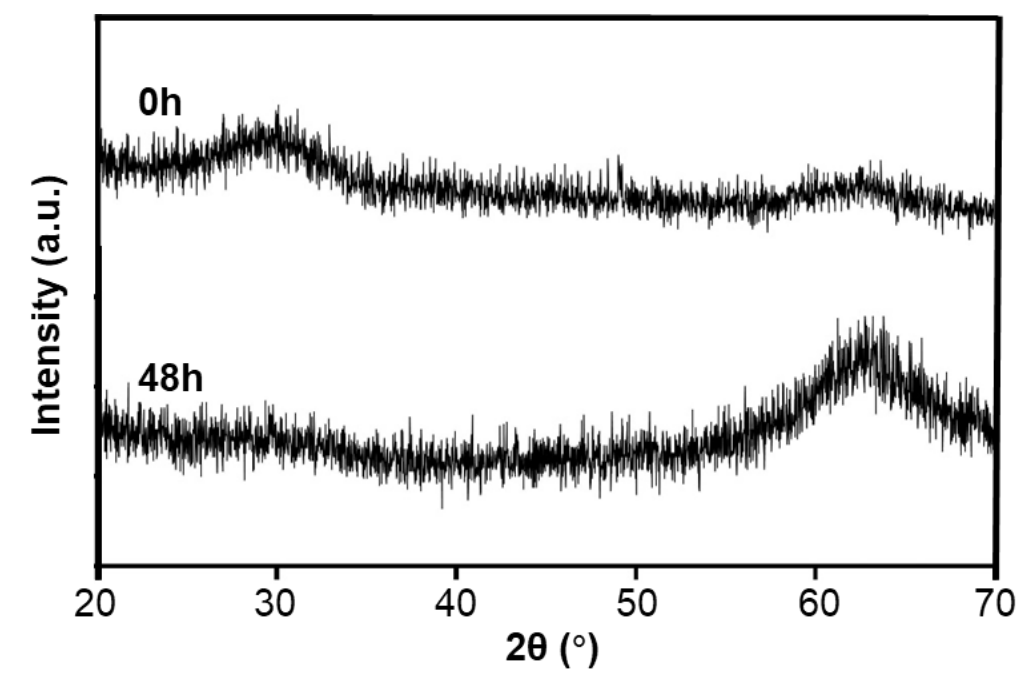

Figure S4. The XRD spectrum of BCP NPs incubated in medium with $10 \%$ serum for $0 \mathrm{~h}$ and $48 \mathrm{~h}$

2. The plasmid DNA loading and release profiles

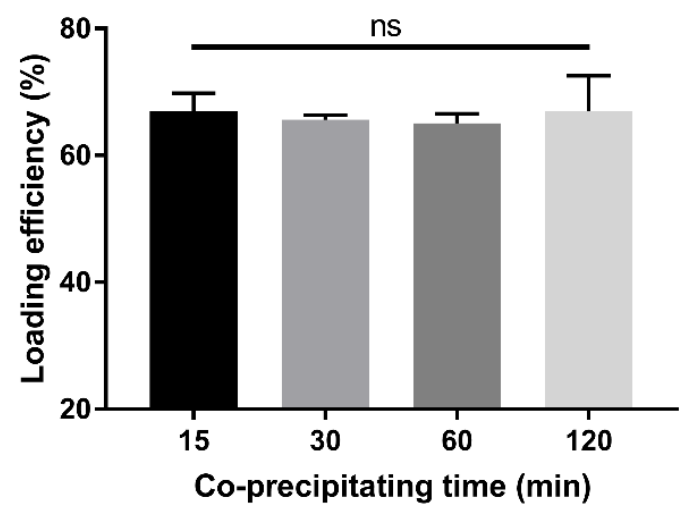

Figure S5. The time-related DNA loading efficiency for 15 to $120 \mathrm{~min}$. 


\section{Endo/lysosomal escape of BCPNPs}

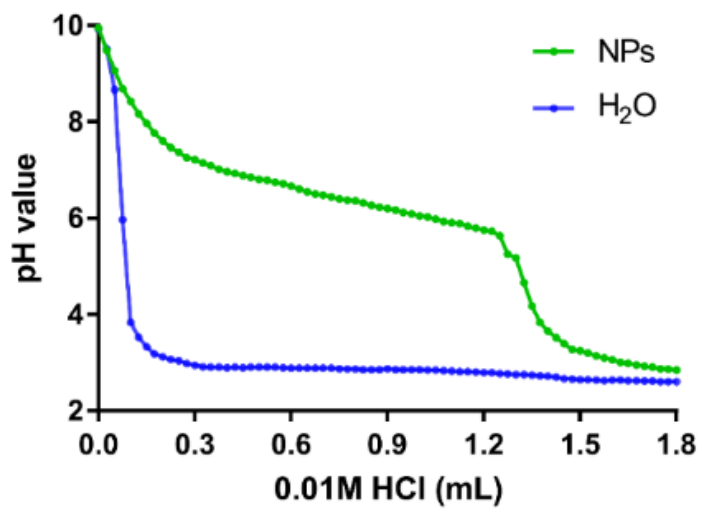

Figure S6. The titration assay of the buffering capacity of BCP NPs.

\section{In vitro transfection of $\mathrm{pEGFP}$ by BCP NPs}
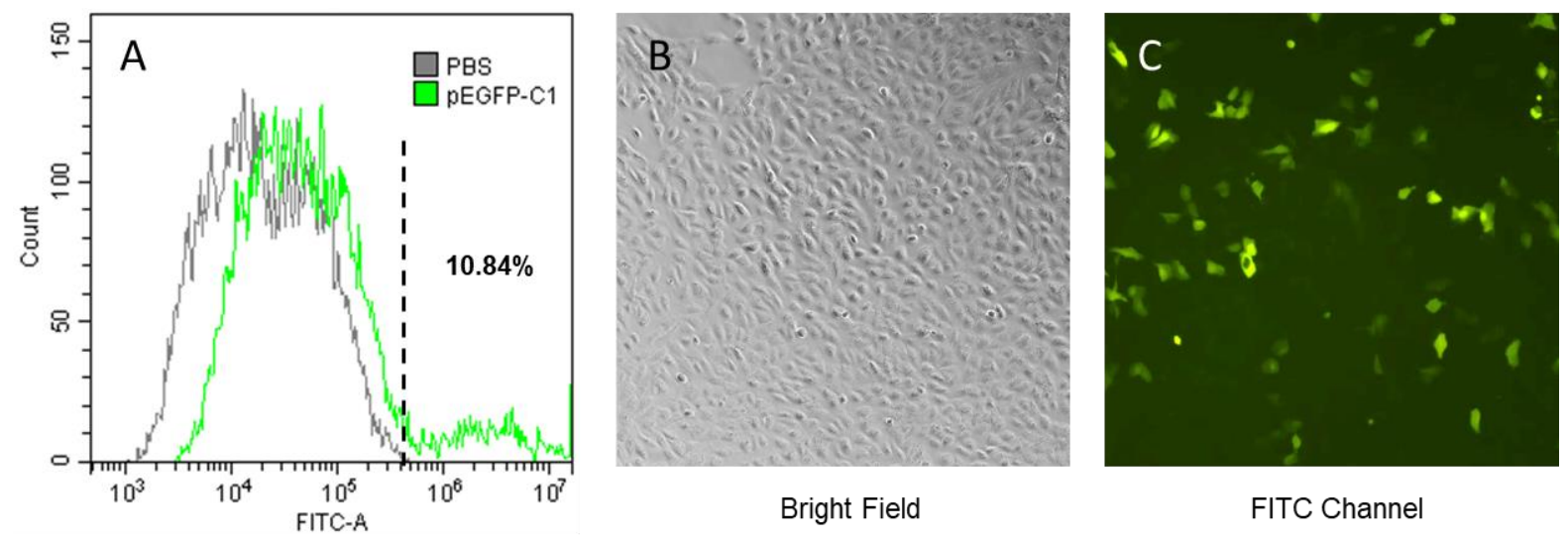

Figure S7. The pEGFP transfection to U2OS cell line. For quality control of pEGFP-C1 plasmid, the

U2OS cell line was used for plasmid transfection. A: the FACS result of pEGFP transfected U2OS cells. B and C: the microscopy images of pEGFP transfected U2OS cells. 

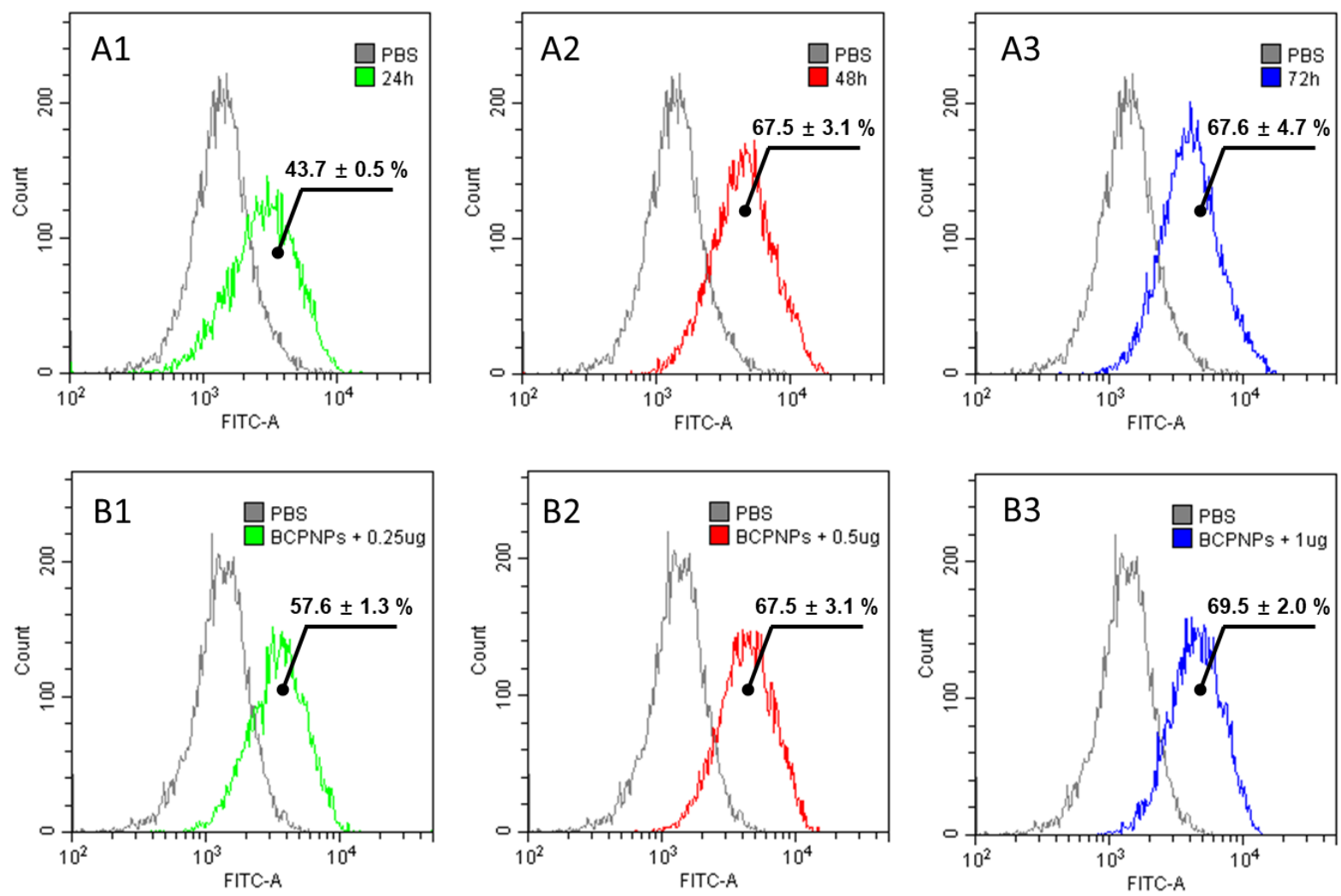

Figure S8. The pEGFP transfection to RAW 264.7 cell line. The FACS results of GPF expression showing by the histogram, which is corresponding to Fig 5. A1 to A3: The time $(24,48$, and $72 \mathrm{~h})$ related GFP expression on RAW 264.7 cell shows by FITC channel fluorescent intensity. B1 to B3: The DNA dose $(0.25,0.5$ and $1 \mu \mathrm{g} /$ well) related GFP expression on RAW 264.7 cell shows by FITC channel fluorescent intensity. 